\title{
Die Reaktionen österreichischer Schriftsteller auf den Kriegseintritt Italiens am Beispiel der D'Annunzio-Rezeption
}

Dir aber wehe, Stampfende Zeit!

Wehe dem scheußlichen Gewitter der eitlen Rede!

(Franz Werfel, Der Krieg

[4. August 1914])

\section{Einleitung}

Mit der Kriegserklärung Italiens an ÖsterreichUngarn endet das, was der bekannte Historiker Manfried Rauchensteiner »[e]ine Geschichte von Fehleinschätzungen und Verwirrspielen, unfreundlicher Nachbarschaft, , Tücke und Treulosigkeit< und >sacro egoismo « " genannt hat. ${ }^{1}$ Damit meint Rauchensteiner in erster Linie die schwankende Haltung, welche die österreichische Regierung nach dem Ausbruch des Ersten Weltkrieges im August 1914 gegenüber der italienischen Neutralität einnahm. Anstatt ernste diplomatische Verhandlungen mit dem noch Verbündeten aufzunehmen, um eventuell die territorialen Angebote der Entente-Mächte zu überbieten (oder auf andere Weise zu kompen-
Die vorliegende Studie analysiert die Reaktionen österreichischer Schriftsteller auf Italiens Kriegseintritt an der Seite der Entente im Mai 1915. Der Fokus liegt auf der D'Annunzio-Rezeption, weil der Autor der Laudi für diesen Schritt Italiens verantwortlich gemacht wird. Alles, was im österreichischen Literaturbetrieb Rang und Namen hat, beteiligt sich an der heftigen Polemik gegen den italienischen Dichter; dabei profiliert sich speziell Stefan Zweig - ein Umstand, der bisher in der Forschung unbeachtet geblieben ist. Die heftige Auseinandersetzung bietet den Autoren der Wiener Moderne die Möglichkeit, mit ihrer bisherigen D'Annunzio-Faszination abzurechnen. 
sieren) und somit Italiens Kriegseintritt zu verhindern, fühlte sich die österreichische Regierung durch die >italienische Krise` überfordert, unterschätzte den Ernst der Lage und setzte bis zum Schluss auf die Taktik des Abwartens und Hinauszögerns. Am 25. Mai 1915 war es dann soweit: Italien versuchte, den Kriegseintritt im Namen des ssacro egoismo < bzw. der Vollendung der Idee des Risorgimento zu rechtfertigen, auf die Kriegserklärung des `unfreundlichen Nachbarn ‘ folgte in Österreich prompt eine Welle der Entrüstung, der Empörung und der Beschimpfungen. Die öffentliche Meinung und die Presse projizierten auf die Italiener eine Reihe von Feindbildern, worunter der Vorwurf von `Tücke und Treulosigkeit`nicht einmal der schlimmste waren.

Parallel dazu brach auch ein »Krieg der Geister «² zwischen Schriftstellern, Intellektuellen und Wissenschaftlern beider Nationen aus. Der unbestreitbare Protagonist dieses Konflikts auf italienischer Seite war Gabriele D’Annunzio, der um die Jahrhundertwende nicht nur der international bekannteste Dichter des italienischen Symbolismus war, sondern sich auch als Prototyp des 'poeta vates $<$ im Sinne eines nationalen Dichters profiliert hatte. In dieser Rolle war er schon sehr früh mit seinen Odi Navali (Schiffsoden, 1906) und dem Drama La nave (Das Schiff, 1908) für ein starkes Italien eingetreten. Parallel dazu hatte er die Österreicher als »Barbaren « hingestellt. ${ }^{3}$ Im Jahre 1912 hatte er die imperialistischen Ambitionen Italiens in Afrika mit der gegen die Türkei gerichteten berühmten Canzone dei Dardanelli unterstützt, die zum Teil zensiert wurde, weil einige Terzinen ÖsterreichUngarn und seinen Kaiser verspotteten. Nach dem Ausbruch des Ersten Weltkrieges im August 1914 hatte D’Annunzio für den Kriegseintritt Italiens aufseiten der Entente plädiert. Der Höhepunkt seiner Kriegspropaganda waren dann zwei Reden im Mai 1915: die erste in Ligurien anlässlich der Einweihung eines Garibaldi-Denkmals und die zweite, kurz darauf, vor der Abgeordnetenkammer in Rom. Italien hatte sich beim geheimen Londoner Vertrag vom 26. April 1915 gegenüber den Mächten der Entente verpflichtet, binnen dreißig Tagen in den Krieg einzutreten, was es dann pünktlich am 25. Mai tat; so hatten D’Annunzios Reden praktisch keine direkte politische Wirkung. Dafür waren sie entscheidend für die Legendenbildung um seine Person bzw. für die Stärkung seiner Rolle als Dichter der Nation - und dies nicht nur in Italien, sondern auch in Österreich-Ungarn. ${ }^{4}$

2 Schneider/Schumann (Hgg.): Krieg der Geister.

3 Vgl. Ritter-Santini: Pari e Impari.

4 Vgl. Lunzer: „O poésie«. 
Daraus folgt, dass D’Annunzio, wie im Folgenden gezeigt werden soll, auch zum wichtigsten polemischen Ziel der Reaktionen der österreichischen Schriftsteller wurde, die mit ihm auch das Herz des >unfreundlichen Nachbarn treffen und diesen bestrafen wollten. Das betrifft auch diejenigen zahlreichen Autoren, die sich früher für ihn eingesetzt hatten. ${ }^{5}$ Dazu gehören etwa Hugo von Hofmannsthal, der ihn den »originellsten Künstler, den Italien augenblicklich besitzt $«{ }^{6}$ genannt hatte. Mit seiner Verehrung für die Schauspielerin Eleonora Duse und seinen Rezensionen der Aufführungen von D’Annunzios Theaterstücken in Österreich hatte auch Hermann Bahr zu dessen Beliebtheit maßgeblich beigetragen. ${ }^{7}$ Das änderte sich schlagartig, als die österreichischen Schriftsteller mit wenigen Ausnahmen ihre Pflicht als Patrioten im >Krieg der Geister zu erfüllen begannen.

Bei der Darstellung der literarischen Reaktionen auf D’Annunzio aus Österreich lege ich zuerst den Schwerpunkt auf Stefan Zweig. Bisher blieb unbeachtet, dass auch ihm D'Annunzio als der wichtigste italienische Dichter der Moderne galt. Während zum Beispiel bekannt ist, dass Hofmannsthal seine anfängliche Zuneigung für D’Annunzio zurücknahm, als sich der italienische Dichter in der Politik engagierte, wusste man bisher kaum, dass der Italiener auch für Zweig zum Stein des Anstoßes wurde, auch wenn Zweig nach scharfer Kritik an dessen politischen Einmischung immer wieder versuchte - anders, als es Hofmannsthal tat - die Bedeutung von D’Annunzio als Dichter nicht aus den Augen zu verlieren. Im zweiten und dritten Teil der vorliegenden Studie wird dann der erwähnte >Krieg der Geister in seiner Mehrdimensionalität untersucht, um dann am Schluss die Folgen des `Falles D’Annunzio`zu erläutern.

\section{Die Polemik gegen D’Annunzio im Vorfeld des Ersten Weltkriegs: das Beispiel Stefan Zweig}

Zwar fängt Zweigs Beschäftigung mit D’Annunzio vor Beginn des Ersten Weltkrieges an und endet lange danach, trotzdem bildet der Krieg den geheimen Angelpunkt seiner Auseinandersetzung mit ihm. Durch Zweigs Verhältnis zu D’Annunzio zieht sich - fast bis zuletzt - ein Widerspruch, der nie versöhnt wird: Der Liebe für den einzigartigen Dichter steht die konsequente Abneigung gegenüber dem spoeta vates` gegenüber. Schon

5 Vgl. Kupka: Der ungeliebte D’Annunzio.

6 Hofmannsthal: D’Annunzio, S. 176. Vgl. Raponi: Hofmannsthal e l'Italia.

7 Vgl. Dangel-Pelloquin: »Mondaine Stimmungsakrobaten«. 
1903 vergleicht Zweig das lyrische Talent von D’Annunzio mit jenem von Stefan George und bewundert die Musikalität seiner Gedichte bzw. deren Fähigkeit, die Welt des Traums zu beschwören. ${ }^{8}$ Der Autor der Laudi gilt ihm als Erneuerer der lyrischen Sprache, weil er einen neuen Rhythmus in die Lyrik eingeführt habe. Als Zweig 1912 zusammen mit dem deutschen Schriftsteller Ernst Lissauer eine internationale Anthologie von Dichtern plant, die sich der freien Rhythmen bedienen, möchte er darin die Stimme von D’Annunzio vertreten sehen. ${ }^{9}$ Der Erste Weltkrieg tut dieser Bewunderung für den italienischen Sprachkünstler keinen Abbruch. Zweig empfiehlt Beispiele aus seiner Prosa zur Veröffentlichung beim Insel-Verlag. Dazu kommt, dass er die italienischen Ausgaben von D’Annunzio wegen ihres bibliophilen Charakters und der Illustrationen von De Carolis bewundert und den Dichter nicht zuletzt als raffinierten Interpreten von Dante hoch schätzt.

Trotzdem mischt sich in die Bewunderung für den italienischen Dichter von Anfang an auch Kritik. In einer Rezension der deutschen Übersetzung von D’Annunzios Römischen Elegien (1904) stellt Zweig den Anspruch des italienischen Dichters in Frage, ein Klassiker der Moderne zu sein und die Nachfolge Goethes in Italien anzutreten ${ }^{10}$ - ein Urteil, das zu revidieren wäre, wenn man bedenkt, dass sich der Italiener mit Romanen wie Il Piacere (Lust, 1888) oder L'innocente (1892) bzw. mit dem Trionfo della morte (Triumph des Todes, 1894) internationale Anerkennung erworben hatte. Es liegt der Verdacht nahe, dass diese Kritik bereits Ausdruck des Unbehagens gegenüber dem wachsenden Engagement von D’Annunzio zugunsten des Irredentismus und des Panitalianismus sei.

Die Einwände gegen D’Annunzio als nationalen Dichter kommen in der Rezension des Dramas La Nave (Das Schiff, 1908) zum Ausdruck. Mit seinem Stück, so Zweig, wollte der italienische Dichter den imperialistischen >Griff nach der Macht Italiens rechtfertigen, der tatsächlich bald darauf in Afrika erfolgen und sich in der Eroberung Libyens im Jahre 1911 konkretisieren sollte. Zweig beschreibt, wie D’Annunzio das eigene Volk aufzufordern versuchte, die alte Hegemonie im Adriaraum wie zur Zeit der Republik Venedig im späten Mittelalter und in der frühen Renaissance zurückzuerobern. Zweig erteilt diesen Forderungen eine Absage und be-

9 Vgl. den Brief von Stefan Zweig an Benno Geiger vom 24. Mai 1912: „Die zweite [Bitte] geht dahin, ob du uns nicht für dieses Buch das erste Gedicht der Laudi von D’Annunzio übertragen könntest, das dieser [der Anthologie] Idee vollen Ausdruck gibt.« Zit. nach: Meli/Ariè Geiger (Hgg.): Benno Geiger, S. 5. 
zeichnet D’Annunzio als `zu spät‘ gekommenen Nationalisten, weil er die authentischen Bedürfnisse des italienischen Volkes falsch eingeschätzt und nicht anerkannt habe, dass die Modernisierungsansprüche des zurückgebliebenen Landes zum Zeitpunkt des Dramas wichtiger seien als der Wunsch nach Lebensraum jenseits der Landesgrenzen. ${ }^{11}$

In einer weiteren Rezension zu den Werken der neusten italienischen Literatur um die Jahrhundertwende setzt Zweig seine Kritik an den nationalistischen Tendenzen von D'Annunzios Werk fort. Er lobt die Romane von Sibilla Aleramo und Giovanni Cena, Una Donna (1906) und Gli ammonitori (1903), weil er darin Beispiele für eine zeitgemäße Literatur erblickt, die einen »europäischen Atem« hat, weil sie aktuelle Themen wie die Emanzipation der Frau und die Industrialisierung behandelt, die weit über die Grenzen Italiens von Bedeutung sind. ${ }^{12}$

Zweigs Ablehnung von D’Annunzio erreicht ihren Höhepunkt nach dem Ausbruch des Krieges Mitte 1914. Zweigs Tagebücher aus den Jahren 1914 und 1915 dokumentieren, mit welcher Sorge der Schriftsteller die Entwicklung der Diskussionen pro und contra Krieg in Italien verfolgt. Der Tenor der Aufzeichnungen aus dieser Zeit ist ziemlich überraschend, weil der kosmopolitisch gebildete Schriftsteller und zukünftige Pazifist hier mit fremdenfeindlichen Stereotypen argumentiert und sich zu chauvinistischen Stellungnahmen hinreißen lässt. Anlässlich von D’Annunzio Rede von Mai 1915 in Quarto schreibt er zum Beispiel: »Die Rede d'Annunzios wird zur Staatsgefahr für uns, die [italienischen] Zeitungen waren so unverschämt, es als `Frechheit $\mathrm{zu}$ empfinden, daß wir ein französisches Kriegsschiff in der Adria in Grund bohrten. ${ }^{13}{ }^{13}$ Zugleich schimpft der sonst zurückhaltende Schriftsteller zornig über die Italiener, weil sie sich in seinen Augen des Verrats gegen die früheren Alliierten des Dreibundes schuldig gemacht hatten:

Dennoch vermöchte ich niemals einem Italiener mehr frei ins Gesicht zu blicken. Sie haben uns zu sehr gequält mit ihrer Perfidie, mit ihrer Verlogenheit, die diesem Raub noch edle Motive unterschiebt. Gegen sie wird sich Deutschlands Haß noch nach Jahrhunderten wenden: es ist eigentlich Wahnsinn, den sie begehen. ${ }^{14}$

Als D’Annunzio am 20. Mai, wenige Tage vor der Kriegserklärung, seine zweite berühmte Rede vor dem italienischen Parlament hält, kommentiert Zweig mit Sarkasmus: »Grenzenloser Jubel für D’Annunzio. Er hat es er-

14 Ebd. Vgl. auch die Notiz vom 11. Mai:»[...] ich kenne die Italiener, die verlogene Ruhmsucht für eine Sache ohne Gefahr.« (ebd., S. 169) 
reicht, l'alta cima, höher als Victor Hugo, als je ein Moderner ist er im Staate gestiegen. Und es kostet 100000 das Leben. ${ }^{15} \mathrm{Im}$ triumphalen Erfolg von D’Annunzio sieht Zweig ein Ereignis von hoher symbolischer Bedeutung: seine offizielle Weihe zum nationalen Dichter. Die Anerkennung und die Ehre, die ihm nun in Italien zuteil werden, sind in seinen Augen noch größer als jene von Victor Hugo in Frankreich. In der Rezension zum Das Schiff hatte Zweig D'Annunzio mit dem französischen Schriftsteller verglichen und letzteren als Vorbild des nationalen Dichters stilisiert, der sich die Forderungen seines Volkes zum richtigen Zeitpunkt zu eigen gemacht und diese vertreten habe, während sich D'Annunzio in seinen Augen für Ansprüche stark gemacht habe, die nicht aktuell oder prioritär gewesen seien. Nun revidiert Zweig seine Meinung und stellt den italienischen Dichter höher als seinen französischen Konkurrenten. Zugleich nennt er aber den Preis eines solchen Erfolges, den das italienische Volk zahlen werde: eine Hekatombe von Soldaten und Zivilisten im Krieg.

Am 25. Mai 1915 erfolgt die offizielle Kriegserklärung Italiens an Österreich-Ungarn. Als Reaktion darauf startet die offizielle Propaganda in Österreich eine mächtige Pressekampagne gegen Italien als Bündnisbrecher und gegen D’Annunzio als Kriegstreiber. Zu diesem Zeitpunkt würde man erwarten, dass sich Zweig aufgrund seiner bisherigen Meinungen daran beteiligt bzw. diese Attacken sogar noch verstärkt. Stattdessen tritt er im Tagebuch aus dem Chor der anti-italienischen Stimmen heraus und nimmt D’Annunzio und die Italiener in Schutz. Er bezeichnet die Vorwürfe der Feigheit gegen das italienische Volk als unangebracht und polemisiert scharf gegen den »intellektuellen Pöbel « der Journalisten, die es gewagt hätten, ihre Stimme gegen D’Annunzio zu erheben, ohne einen Funken seines poetischen Talents zu besitzen. ${ }^{16}$ Später kommentiert Zweig D’Annunzios berühmten Flug über Wien am 8. August 1918 mit keinem Wort.

\section{D’Annunzio als Ziel der Angriffe österreichischer Schriftsteller in den Kriegsjahren 1915 und 1916}

Die kritischen Reaktionen der österreichischen Schriftsteller gegen D’Annunzio lassen sich in mindestens zwei große Phasen gliedern: Unmittelbar nach der Kriegserklärung gibt es aufgeregte, empörte Stellungnahmen, in denen D’Annunzio als Quintessenz der negativen Attribute des italienischen 
Volkes diffamiert wird. Die Feindbilder, die gegen das Nachbarvolk aktiviert werden, kreisen um dessen angeblichen Hang zu Verrat, Ruhmsucht und Feigheit. Danach folgt eine zweite Phase der Auseinandersetzung, in der neben der direkten Polemik die Persiflage dominant wird. Dabei ist es auffallend, dass sich fast alles, was in Österreichs Literatur Rang und Namen hat, an dieser Kampagne gegen den italienischen Dichter beteiligt.

Die Tagebücher Arthur Schnitzlers enthalten im ersten Kriegsjahr keine ausführlichen Kommentare zu den politischen Entwicklungen in Italien wie jene Zweigs. ${ }^{17}$ Er verwendet in seinem Brief vom 22. Mai 1915 an den Verleger Samuel Fischer harte Töne:

Wenn dieser Brief in Ihre Hände kommt, haben die Feindseligkeiten mit Italien wohl schon in aller Form ihren Anfang genommen. Wie ich eben versuche mehr dazu zu sagen, fühle ich zugleich, daß ich darauf verzichten muß, da der deutsche Sprachgeist das Wort noch nicht gefunden hat, in dem die Begriffe Verrat, Erpressung, Tücke, Verlogenheit und d'Annunzio sich vereinigt ausdrücken ließen. ${ }^{18}$

Der Name D’Annunzio ist im Zitat austauschbar mit vielen negativen Merkmalzuschreibungen an Italien, die auch Zweig genannt hat. Daraufhin nimmt Fischer die Bücher D’Annunzios aus dem Verlagsprogramm.

Auch Alfred Polgar reagiert auf Italiens Kriegseintritt sofort mit einem Angriff auf den italienischen Dichter. Fünf Tage nach der Kriegserklärung, am 30. Mai, verfasst er einen Essay mit dem Titel d'Annunzio. Darin beschreibt er den italienischen Dichter als Volkstribun, als großen Verführer und Demagogen, der das italienische Volk dazu gebracht habe, »Wünsche mit Wirklichkeiten ${ }^{19}{ }^{\mathrm{zu}}$ verwechseln (es sei eine Illusion, den Krieg gegen Österreich-Ungarn zu gewinnen). Polgar stellt fest, dass D'Annunzio diese Verführungskraft ausgeübt habe, ohne auf seine ornamentale und künstlich überladene Sprache zu verzichten. Er nennt diese Sprache »einen prunkvollen Schwall, der seine [D’Annunzios] Bücher ungenießbar « mache. ${ }^{20}$ Auf der anderen Seite kann er nicht umhin, die große "Massenwirkung" von D’Annunzios Rhetorik anzuerkennen. Bei aller Kritik an seiner künstlichen Sprache attestiert er die Modernität seiner rhetorischen Strategien und Inszenierungstechniken sowie seine Fähigkeit, die Massen zu verführen. Dass D’Annunzio ein Meister der Massenmanipulation war, wurde den meisten

17 Einmal ist nach der ersten erwähnte Rede von D’Annunzio in Quarto der lakonische Satz am 6. Mai 1915 zu lesen: »Italien höchst bedenklich, d’Annunzios Rede.« (Schnitzler: Tagebuch, S. 195)

18 Schnitzler: Briefe 1913-1931, S. 88.

19 Polgar: d'Annunzio, S. 6.

20 Ebd. 
erst nach dem Fiume-Unternehmen 1919 richtig bewusst. ${ }^{21}$ Polgar erkennt es schon 1915. Ihn interessiert besonders die Frage, warum ein Ästhet wie D’Annunzio seinen Elfenbeinturm plötzlich verlassen will, um die Gunst des `profanum vulgus zu erobern, die er bis dahin verachtet hatte. Er glaubt ein Ungenügen an der rein ästhetischen Existenz zu erkennen sowie den Wunsch, die Kunst ins Leben zu überführen. Polgar möchte D’Annunzio als Lügner entlarven, daher unterstellt er seinen Reden das Fehlen jeder echten Leidenschaft. D’Annunzio habe den Kontakt zum Volk auf Kosten der Wahrheit hergestellt. Er bedauert die Italiener, die in seinen Augen bald merken sollen, welchen Fehler sie begangen hätten, indem sie D'Annunzio gefolgt seien - eine Prophezeiung über die Zukunft des Landes, wie sie bereits Zweig verkündet hatte.

Vom Fall D’Annunzios ausgehend stellt Polgar dann die grundsätzliche Frage, ob es legitim sein, dass »ein Dichter, ein Kulturmensch, ein Geistesaristokrat ${ }^{22}$ für den Krieg predige. Er beantwortet die Frage mit der Unterscheidung zwischen gerechtem und ungerechtem Krieg. Das heißt: Er verurteilt D’Annunzio als »Rattenfänger, der die Kindlichen und Vertrauensvollen und Neugierigen ins Grab flötet«, hingegen verteidigt er die Intellektuellen, die den Krieg preisen, wenn dieser als »Heimsuchung über ein Volk fällt «. ${ }^{23}$ Am Schluss des Textes bezeichnet er allerdings den Krieg als große Katastrophe der Menschheit.

Dabei fällt auf, dass Polgars Sprache mitten in einem Klima extremer Aggressivität von jeder Form von Chauvinismus frei ist. Die Art und Weise, wie er trotz der Animosität gegen D’Annunzio über die Italiener spricht, unterscheidet sich grundsätzlich von jener in den Tagebüchern von Schnitzler und Zweig. Während diese Hasstiraden gegen die früheren Verbündeten niederschreiben, stellt Polgar die Italiener als unkritische Opfer eines klugen Demagogen dar und nennt sie in erster Linie `vertrauensvolk und `neugierig‘. Im Gegensatz zu Zweig und Schnitzler bedauert er das tragische Schicksal, das für sie vorgesehen ist, und stellt sich das Moment ihrer Desillusionierung vor: „Wenn es [das Volk] in Tagen der Trauer und Not nach den goldenen Worten seines Dichters greifen wird, wird ihm Stroh und Häcksel in den Händen bleiben. $\ll^{24}$

Die Attacken gegen den italienischen Dichter und die Italiener erreichen einen weiteren Höhepunkt in dem Essay Von Dante zu d'Annunzio

21 Vgl. dazu Brittnacher: „Der Rhythmus hat immer recht«; Gumbrecht/Kittler/Siegert (Hgg.): Der Dichter als Kommandant.

22 Polgar: d'Annunzio, S. 8.

23 Ebd., S. 6.

24 Ebd., S. 8. 
von Egon Friedell, das dem gleichnamigen Pamphlet von 1915 den Titel gibt. Darin verfolgt Friedell das Ziel, die Italiener gegenüber den anderen Kulturvölkern völlig zu diskreditieren. Friedell geht von der Voraussetzung aus, dass die Italiener weniger "Leistungen von europäischem Ruf « als die Nachbarvölker hervorgebracht haben, und fragt provokant: "Warum schickt Italien über seine Grenzen nur Schwindler und Flachköpfe? «, um selbst zu antworten: »Weil es nichts anderes hat. $\aleph^{25}$ Als Beleg dafür führt er die angebliche Überlegenheit von Theodor Mommsens Darstellung von Julius Caesar über jene von Guglielmo Ferrero $a^{26}$ - eine Überlegenheit, die sich in seinen Augen auch auf dem Gebiet der »italienische[n] Schauspielkunst « ${ }^{27}$ beobachten lasse, wo Schauspieler wie Ermete Zacconi und Ermete Novelli zu wünschen übrig ließen. Im wissenschaftlichen Bereich gehe den Italiener nicht besser. Cesare Lombrosos Werk Genie und Irrsinn (1887) führe in den Augen von Friedell nur eines vor: »Der Gott Italiens ist der Unsinn. ${ }^{28}$

Im zentralen Teil des Essays stellt Friedell Dante D’Annunzio gegenüber und vertritt die Überzeugung, dass der Weg Italiens "von Dante zu D’Annunzio« der Weg eines katastrophalen und irreversiblen Verfalls sei. Er stellt D’Annunzios Anspruch in Frage, sich als Nachfolger von Dante zu empfinden, zumal der Autor der Commedia nur bedingt als Italiener bezeichnet werden dürfe: »Dieser große Longobarde [sic] steht seit sechshundert Jahren über Italien als ein brennendes Warnungszeichen, ein Zeichen dessen, was der italienische Genius hätte werden können und wovon er das Gegenteil geworden ist. ${ }^{29}$ Friedell argumentiert hier sehr ähnlich wie Hofmannsthal in seiner Antwort auf D'Annunzios neunte Canzone: Hatte dieser zwischen dem »falschen « Italien D’Annunzios und dem authentischen der großen Kunst (Mantegna) bzw. der großen Politik (Mazzini) scharf unterschieden, ${ }^{30}$ so trennt jetzt Friedell dezidiert zwischen Dantes Italien und jenem von D’Annunzio. Selbst »die große und reine Kunst« von Eleonora Duse ist für Friedell "gewiß nicht italienisch «: ${ }^{31}$ Sie entspringe "aus ihrer Weiblichkeit: Sie ist daher etwas ganz Internationales. ${ }^{32}$

Friedell: Von Dante zu d'Annunzio, S. 55.

Polgar bezieht sich auf Theodor Mommsens Römische Geschichte (1854-1856) und Guglielmo Ferreros Größe und Niedergang Roms.

Friedell: Von Dante zu d'Annunzio, S. 56.

Ebd., S. 58.

Ebd.

Hofmannsthal: Antwort auf die »neunte Canzone".

Friedell: Von Dante zu d'Annunzio, S. 56.

Ebd., S. 57. 
In diesem Essay erscheint D’Annunzio wieder als Quintessenz der italienischen $>$ Seele $\prec$ im negativen Sinne - als Verkörperung des $>$ Renaissancemenschen . Durch diese Annahme will Friedell nicht so sehr darauf hinweisen, dass mehrere Werke von D'Annunzio in der Zeit der Renaissance spielen oder dass sich der italienische Dichter gerne als Renaissancefürst inszeniert hat, sondern vielmehr die negativen Merkmale akzentuieren, die mit diesem Topos verbunden sind: »Und hat D’Annunzio vergessen, wen im Inferno der allerentsetzlichste Fluch trifft? Die Verräter! In der äußersten Ferne von Gott zu sein ist ihre Strafe. Im ewigen Eis stecken sie, wo selbst

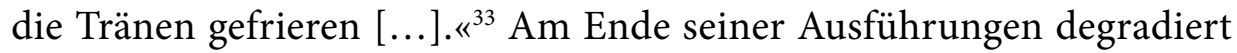
er D’Annunzio sogar zu »einem verkommenen Friseurgehilfen, für den das deutsche Wort >Laffe $<$ beinahe wie eigens erfunden erscheint, und der nun, durch entsprechende Trinkgelder angefeuert, fingerfertig mit seinen ranzigen Pomadentöpfen hantiert. ${ }^{34}$ Hier greift Friedell offensichtlich Stereotypen vom italienischen Barbier bzw. vom Figaro wieder auf.

Nach der ersten Phase der Empörung und der Schimpftiraden wird D’Annunzio allmählich Ziel einer Kampagne der Kriegspropaganda, die im Jahr 1916 kulminiert. In diesem Zusammenhang ist zum Beispiel das Buch Die Front im Tirol von Franz Karl Ginzkey zu nennen, einem guten Freund von Stefan Zweig, der dem bekannteren Schriftstellerkollegen zu einer Stelle im Kriegspressearchiv verholfen hatte. Hier verfassen beide Zeitungsartikel und Bücher, die das Heldentum der österreichischen Soldaten preisen sollen, bis Zweig allmählich zum Pazifismus findet und 1917 in die Schweiz auswandert. Im genannten Buch baut Ginzkey einen scharfen Gegensatz zwischen einem einfachen Tiroler Soldaten und dem italienischen spoeta vates` auf, um die Tugenden des ersteren hervorzuheben:

Das klare, erdgetreue Wesen dieses stolzbescheidenen Sängers, der seinem Volke todesmutig auch zur Tat voraneilt, sticht wunderbar bezeichnend gegen seinen ungleich berühmteren Kollegen im feindlichen Lager und dessen bombastisch in sich selbst erhitzte Phrasen ab. Sitzt auch Herr Gabriele d'Annunzio bereits bei seinen Alpini droben auf irgendeinem umschossenen Felsengrat? So zwischen zwei- und dreitausend Meter, zur Rechten den Gletscher -, zur Linken den Kugeltod? Er könnte dort mithelfen, die Suppe auslöffeln, die er seinem verirrten Volke eingebrockt. Aber die Macht seiner wohlgedrechselten Rede hätte dort droben im Angesicht der schweigenden Höhen wenig Gewalt. Seine Leute würden ihm sagen: Mann, gib uns die Tat und entschäle den Kern deines Denkens. Das Spiel der Begriffe verliert hier jeglichen Wert. ${ }^{35}$ 
In den beiden Fällen, die Ginzkey miteinander vergleicht, haben wir es mit einem Sänger zu tun: Während aber der Tiroler Soldat in seiner Bescheidenheit ein einfaches Kriegslied anstimmt, erscheint der italienische Dichter als perfider Verführer, der seine Redekunst geschickt einsetzt, um ein »verirrtes Volk « zu einem desaströsen Unterfangen zu überreden. In der Realität des Kampfeinsatzes erweise sich jedoch D’Annunzios "Spiel der Begriffe «, so der zweite Teil der Argumentation, als nutzlos: [I]m Angesicht der schweigenden Höhen« der Tiroler Berge zähle nur der österreichische Soldat mit seinem Mut. Vom italienischen Dichter nimmt Ginzkey an, dass er seine Alpini im entscheidenden Moment allein lasse - eine Vermutung, die in dieser Form sicher nicht zutrifft, wenn man die Kampfeinsätze D’Annunzios berücksichtigt: von der so genannten >Beffa di Buccari $<$ bis hin zum Flug über Wien.

Den Topos von D’Annunzio als Verführer der Massen greift in seinen Aphorismen auch Peter Altenberg auf, der sich auf die Pflicht des authentischen Schriftstellers beruft, moralische Verantwortung für die als unmündig bezeichneten Zuhörer zu tragen:

Wer die unglückselig-stupide Menge, diese »Unmündigen des Daseins«, diese »Kleinkinderveranstalter des Daseins« aufwiegelt, irreführt, mit »blechernen Worten« hypnotisiert, ist ein feiger Schandbube, kein Dichter! Kindliche hat man zu unterweisen, nicht rebellisch zu machen, nicht daß sie den Kopf verlieren, den sie überhaupt gar nicht haben. ${ }^{36}$

Altenberg akzentuiert hier den von mehreren Seiten bereits geäußerten Vorwurf der Feigheit gegen D'Annunzio und spricht ihm den Rang des Dichters ab - nicht zuletzt, weil er davon ausgeht, dass sich ein Dichter von der Politik fern halten sollte. Zu diesem radikalen Schluss war bisher keiner seiner Schriftstellerkollegen gekommen.

Während Ginzkey auf den rhetorischen Ernst kriegspropagandistischer Muster setzt, bedient sich Robert Musil - der seinen Kriegsdienst in Tirol absolviert - der subtilen Mittel der Ironie. Als Redakteur der Tiroler Soldaten-Zeitung mit Sitz in Bozen veröffentlicht Musil, der D’Annunzio seit seiner Jugendzeit kannte, im Juli 1916 in seinem Organ eine bissige Persiflage des Berliner Kritikers Alfred Kerr mit dem Titel Ode VI:

I. Un anno dura la guerra Sul commando dell'Inghilterra.

Celebramo questa festa In alle mesta.

II. Nebblich, siamo nella tinta Lo commo dahinter 
O Havanna! O Trabucco!

Non avanti, mà zarucco.

Che triste jubilee

Eiweo, eiweo!

Grande italiana ritirata

In Gorgonzola una mada.

III. La stupidità è il nostro manco!

Tirolo, Gorizia, tutta la somma

Era offerta gratis e franco -

Hemma si gnumma!!

O Tiziano! O Raffaello!

Che slamassello!

O Giulia! Ada! Ida!

Machma frieda! Machma frieda! ${ }^{37}$

Innerhalb der Polemik gegen D’Annunzio bilden die Persiflage und die Karikatur eine eigene Gattung, über Österreich hinaus auch in Italien und Frankreich. ${ }^{38}$ Der wichtigste Beitrag zu dieser speziellen Gattung in der österreichischen Literatur ist jener Franz Bleis, der in seinem Bestiarium der modernen Literatur (1920) D'Annunzio mit einem Pegasus vergleicht ein Hinweis auf die bereits vor dem Ausbruch des Krieges von Franz Kafka registrierte große Leidenschaft des italienischen Dichters für das Fliegen ${ }^{39}$ und möglicherweise auch auf seinen spektakulären Flug über Wien vom August 1918:

Der Pegasus d'Annunzio schlug mit seinen eleganten Hufen die herrlichsten, herrischesten Takte der letzten drei Jahrzehnte, ihm darin gleich nur des Northumberlandhirsches Swinburne Flug und Fougue. Später dann verlangte die Zeit Probe aufs große Wort, und der Pegasus gab sie. Er ließ sich die Hufe mit Eisen beschlagen, wirbelte damit die Trommel und wieherte Fanfaren. Die an tönenden Worten reichste Zeit, die des Krieges und seines Après, machte aus dem Pegasus nicht den Tyrtaios, aber das lauthinwiehernde Schlachtpferd gab den hellen italienischen Trompeten Brust, Luft und Schwung. Ein römischer Kaiser hat sein Leibpferd zum Konsul gemacht - der Pegasus d'Annunzio konnte es für möglich halten, daß ihn sein Volk zum Kaiser der Adria erhebe. ${ }^{40}$

Blei ironisiert D’Annunzios Selbststilisierung zum nationalen Barden Italiens und seine propagandistische Tätigkeit, ohne ihm allerdings wie Peter Altenberg den europäischen Rang strittig zu machen, den er als Dichter in

37 Gottlieb (=Alfred Kerr): Ode VI (zit. nach. Corino: Robert Musil und Italien, S. 40).

38 Vgl. Gec: D’Annunzio nella caricatura mondiale.

39 Vgl. Demetz: Die Flugschau von Brescia. Das Buch beschreibt die Flugschau von Brescia im September 1909. Felix Philipp Ingold bezeichnet Kafkas Prosa-Miniatur Die Aeroplane in Brescia als »eine Charakterskizze von karikaturesker Treffsicherheit« (Ingold: Literatur und Aviatik, S. 55). 
seinen Augen erreicht hat. In Bleis Ironie schwingt der Vorwurf der Hybris mit. Die bereits von Hofmannsthal, Zweig und anderen aufgeworfene Frage nach der Opportunität der Involvierung des Dichters in die Politik wird die Auseinandersetzung um D’Annunzio auch in den zwanziger und dreißiger Jahren bestimmen.

\section{Schlussbetrachtungen}

Aus den vorgestellten Essays und Texten geht deutlich hervor, dass die ersten Reaktionen auf Italiens Kriegseintritt keine sachliche Untersuchung der Gründe enthalten, die zu der bellizistischen Auseinandersetzung zwischen den beiden früher verbündeten Staaten geführt haben. Wie die Polemik gegen D'Annunzio vor Augen führt, werden stattdessen in erster Linie Emotionen mobilisiert, Feindbilder von Italien ins Spiel gebracht, negative Stereotypen zitiert, nationalistische bzw. chauvinistische Parolen angewendet, um den Feind und seinen Barden dem Spott preiszugeben und Hass gegen sie zu schüren. Nur in einigen Fällen mildert die Ironie die Schärfe dieser Attacken.

Ein passender Kommentar zu den polemischen Stellungnahmen zu D'Annunzio und den Italienern ist jenen Passagen der Letzten Tage der Menschheit zu entnehmen, in denen Karl Kraus den Chauvinismus von zwei an der Isonzo-Front kämpfenden österreichischen Offizieren kritisiert. Schon ihre Namen - sie heißen Fallota und Beinsteller - sprechen für sich und sind ein Hinweis auf ihr intellektuelles Niveau. Kraus lässt in einem von beiden Offizieren gesungenen Lied alle Stereotypen der antiitalienischen Propaganda zu Wort kommen (III Akt, 3. Szene):

Wir haben sie guat getroff'n

Die andern dö san gloff'n.

Tschiff, tscheff, tauch, der Wallisch liegt am Bauch.

Könnan nimma Katzl mach'n,

Es tuat halt gar zviel krach'n.

Tschiff -

Den Annunzio und Sonnino

Den machma a no hino.

Tschiff $-[\ldots]^{41}$ 
Indem Kraus eine tragende Säule der Monarchie wie die Offizierskaste offen an den Pranger stellt und ihre Vertreter lächerlich macht, lanciert er eine Attacke an die Monarchie als Ganzes. Parallel dazu stellt Kraus in seinem Drama die Brutalisierung des Menschen im Kriege dar. An anderen Episoden, die Italien zum Thema haben, zeigt er den markanten Unterschied zwischen dem Verhalten des gleichen Menschen im Krieg und im Frieden - zum Beispiel, als der Fregattenleutnant berichtet, das gleiche Venedig bombardiert zu haben, das er in Friedenszeiten als Tourist geliebt hatte (II. Akt, 30. Szene).

In manchen Fällen greift Kraus auch seine Schriftstellerkollegen direkt an. So legt er zum Beispiel schon im Vorfeld des Krieges einige Schwachstellen der Antwort von Hofmannsthal auf D'Annunzios neunte Canzone bloß. Er ironisiert Hofmannsthals Anspruch, sich als Italiener zu empfinden, um selbst Teil des authentischen Italiens zu sein, dem D'Annunzio nach seinem politischen Engagement in seinen Augen nicht mehr angehört: »Herr d'Annunzio aber ist nicht italienisch. Herr Hofmannsthal weiß wenigstens, was italienisch ist. Vielleicht ist er aber sogar italienisch [...]. Vielleicht ist Herr Hofmannsthal gar der d'Annunzio und der d'Annunzio nur ein Hofmannsthal. $\aleph^{42}$ Kraus' Argumente gegen Hofmannsthals Anmaßung ließen sich mutatis mutandis auch auf Friedells Interpretation von Dante als >Langobarden`, der Duse als ıinternationaler Schauspielerin oder auf den >deutschen Dante< von Rudolf Borchardt anwenden. ${ }^{43}$

Obwohl Kraus mit seiner Entlarvung der Kriegspropaganda in Die letzten Tage der Menschheit auch die Grundvoraussetzung für die allgemein verbreitete Polemik gegen D’Annunzio in Frage stellt, lässt sich das bemerkenswerte Phänomen beobachten, dass einige Wortführer dieser Polemik mit ihm am gleichen Strang ziehen, wenn es darum geht, gegen die Presse und ihre Entgleisungen anzutreten. Zum Beispiel spricht Zweig diesbezüglich vom »Journalistischen Pöbel «, ${ }_{44}^{4}$ Polgar hingegen nennt die Journalisten "Brüder im Ungeiste von D’Annunzio « ${ }^{45}$ weil sie das Echo seiner demagogischen Reden vervielfachen. Zweig und Polgar übernehmen den Affekt gegen die Presse zwar von Karl Kraus, schreiben ihm aber unterschiedliche Funktionen zu: einmal soll er auf das Phänomen des allgemeinen Sprachverfalls hinweisen, ein anderes Mal als Kritik an der Demagogie dienen; einmal zugunsten des italienischen Dichters, das andere Mal gegen ihn. 
In den zwanziger Jahren erweitert sich die Diskussion um D’Annunzio im Zusammenhang mit seiner offen zur Schau gestellten Unterstützung für das Mussolini-Regime. Zwölf Jahre nach seiner ersten Wortmeldung kommt etwa Alfred Polgar wieder auf ihn zu sprechen - in einem Essay mit dem Titel Paderewski. D’Annunzio (1927). Inzwischen ist der Krieg zu Ende gegangen, das Fiume-Unternehmen hat stattgefunden, und D'Annunzio lässt sich an der Seite von Mussolini feiern. Indessen hat Polgar seinen Blick auf das D’Annunzio-Phänomen geändert: Während er 1915 den italienischen Dichter noch als absolutes Novum der Kulturgeschichte bezeichnet hatte, betrachtet er jetzt D'Annunzio gemeinsam mit einem weiteren nationalistischen Künstler, dem Polen Paderewski. Von den beiden sagt Polgar: »Sie waren Künstler und wurden `Staatsmänner`. Sie gehörten sich und gehören jetzt der Allgemeinheit. Sie vertauschten ihren Ruhm gegen Popularität. Sie geben das sonderbar parodistische Schauspiel einer Rückbildung Freier zur Unfreiheit. $\ll^{46}$

Polgar kritisiert hier ähnlich wie Zweig den Intellektuellen, der sich in die Politik einmischt. Für ihn sei diese Involvierung in die Politik ein reines Verlustgeschäft: er büße seine Freiheit und seine Geltung als Künstler ein; dafür, fügt Polgar sarkastisch hinzu, gewinne er eine Uniform. Aber wichtiger noch ist für den österreichischen Kritiker der Umstand, dass D'Annunzio insgesamt nicht mehr als Einzelfall zu sehen ist: Er sieht darin ein europäisches Problem.

Im gleichen Jahr wie Polgars Essay erscheint auch das berühmte Buch von Julien Benda über den Verrat der Intellektuellen. Benda kritisiert D'Annunzio als typisches Beispiel für den Verrat der allgemeinen Werte der Aufklärung, die ein Intellektueller, welcher Couleur auch immer, welcher Nationalität auch immer, verteidigen sollte. Der Franzose erwähnt das Drama Das Schiff als Beleg für die nationalistischen Entgleisungen des italienischen Dichters, ähnlich wie es schon Stefan Zweig getan hatte, und nennt es ein Novum in der Geschichte der ১Clercs‘. Wie immer man $\mathrm{zu}$ seinen Thesen steht - mit seinem Buch liegt Benda auf derselben Linie wie Polgar: Das Problem des Verrats der Intellektuellen sei kein nationales, sondern ein europäisches Problem. ${ }^{47}$

Im Gegensatz zu diesen Stellungnahmen vertritt Stefan Zweig in den zwanziger Jahren eine positive Haltung gegenüber D’Annunzio. Während Polgar angesichts der Radikalisierung der politischen Konfrontation in Europa den italienischen Dichter nur als nationalen Barden betrachtet, besinnt

47 Vgl. Lunzer, Zum Thema »trahison des clercs«. 
sich Stefan Zweig nach dem Krieg auf seine frühere Bewunderung für ihn und verzichtet auf den ideologischen Verdacht gegen das frühere Vorbild. Im Rahmen einer Umfrage der italienischen Zeitschrift »Leonardo« von 1925 bezeichnet er D'Annunzio als einen »leidenschaftlichen Wortbildner, als glühenden Künstler, der von sich selbst und von allen Formen der Schönheit berauscht ist $«{ }^{48}$ Mit dieser Definition D’Annunzios als Genie, das sich dem Kult der Schönheit verschrieben hat, sieht Zweig von moralischen Kategorien völlig ab und rückt den italienischen Dichter in die Nähe eines dämonischen Künstlers. 1925 veröffentlicht er ein Buch, in dem er eine Poetik des Dämonischen anhand der Beispiele Hölderlin, Kleist und Nietzsche erläutert. ${ }^{49} \mathrm{Im}$ Zentrum dieser Kunstauffassung stehen Begriffe wie Kreativität, Fanatismus, Heroismus und auch Tragik. Es stellt sich die Frage, ob die Bezeichnung von D’Annunzio als dämonischer Künstler für den italienischen Dichter zutrifft oder nicht. Sicher ist, dass Zweig hier seine Forderung eines Zusammengehens von Literatur und Moral revidiert, die er 1914 in Anschluss an seine Kritik an D’Annunzio und Verhaeren gestellt hatte. ${ }^{50}$ Damals hatte er die Auffassung vertreten, dass eine Literatur, die sich schöner Worte bediene, um moralisch bedenkliche Inhalte zu vertreten, ihre ganze Substanzlosigkeit an den Tag lege. ${ }^{51}$ Zehn Jahre später spielt diese Position keine Rolle mehr.

Am Beispiel von Polgar und Zweig lassen sich zwei grundsätzliche Positionen gegenüber D’Annunzio verdeutlichen: Während die einen Autoren einen ideologischen Verdacht gegen D’Annunzio hegen, ihn zum chauvinistischen Dichter bzw. zum Vorläufer des Faschismus abstempeln, versuchen die anderen zwischen seiner poetischen Leistung und seiner politischen Rolle zu unterscheiden, um die eine unabhängig von der anderen würdigen zu können. Der Intensitätsgrad der Auseinandersetzung ist dabei der Bedeutung von D’Annunzio als literarisches Vorbild für die einzelnen Schriftsteller angemessen.

$\mathrm{Zu}$ den diesbezüglich wichtigsten Autoren gehört neben Polgar und Hofmannsthal Robert Musil, der in einem Rückblick auf sein frühes Interesse für D’Annunzio anlässlich des Todes des Dichters am 2. März 1938 und kurz nach dem `Anschluss $<$ bemerkt: »Er war soweit ein guter Patriot, als er ein guter Dichter war. Man kehrt das aber um: Er war ein guter Dichter,

49 Vgl. Zweig: Der Kampf mit dem Dämon. Vgl. auch Birk/Eicher (Hgg.): Stefan Zweig und das Dämonische.

50 Rolland/Zweig: Briefwechsel 1910-1940, S. 124.

51 Ebd. 
weil er ein guter Patriot war. ${ }^{52}$ Am gleichen Tag verfasst Franz Werfel eine Tagebuchaufzeichnung, in der er ebenfalls D’Annunzios Zeit als 'poeta vates` während des Ersten Weltkriegs in Erinnerung ruft und seine poetische Leistung hinter dem politischem Engagement bzw. der Ruhmsucht verschwinden lässt:

Alle seine Masken verschwimmen in die letzte, die Totenmaske. Er war ein großer Dichter, doch hat er das Dichtertum nicht erhöht. Er unterordnete den Dichter im Range dem politischen, dem militärischen Führer, ja dem nationalen Filibustier. Das geschah, weil sein Ehrgeiz so ungeheuerlich [war], das ihm kein Ruhm genügte, nicht die stille, echte, beständige Ehre, die einzig und allein das geistige Sein und Schaffen zu vergeben hat. Er betete die Macht an und den grellen Erfolg sehr kühner Abenteuer, die er mit großem Mute unternahm, teils aus wirklichem Fanatismus, teils um sich dadurch zum nationalen Heros heraufzunumerieren. ${ }^{53}$

Andere Autoren hingegen haben weniger Berührungsängste mit dem italienischen Dichter und sind der Meinung, dass man D’Annunzios Beitrag zur Moderne retten könne, auch wenn er politisch nicht zu tragen sei. Das ist die Position von Stefan Zweig, der die Gedichte D’Annunzios 1934 ins Exil mitnimmt, obwohl er dessen Schulterschluss mit Mussolini aufs schärfste verurteilt hat. ${ }^{54}$

Ähnlich verhalten sich Walter Benjamin und Bertolt Brecht. Nachdem Benjamin während seines Aufenthalts auf Capri 1916 die Laudi und Merope gelesen hat, ist er gerne bereit, für die Zeitschrift Der Querschnitt

52 Musil: Tagebücher, Aphorismen, Essays und Reden, S. 142. Musil distanziert sich von seiner früheren Bewunderung für den Roman Il piacere (Lust): »Es ist eines der ersten Bücher gewesen, durch die ich vor 40 Jahren Bekanntschaft mit der $>$ Moderne machte, und eins der ersten, die Einfluß auf mich hatten. Ich gäbe etwas darum, noch zu wissen, welchen. Wahrscheinlich eine allgemeine Immoralität und ebenso allgemeinen Ästhetizismus.« (ebd., S. 470)

53 Werfel: Zwischen Oben und Unten, S. 455.

54 Als Zweig 1929 in der Nähe der von den Carabinieri bewachten Villa D’Annunzios am Gardasee vorbeikommt, in der der italienische Dichter nun zurückgezogen lebt, formuliert er seinem Freund Romain Rolland gegenüber ein sehr hartes Urteil über den >poeta vates؛: »Ich war zu meiner Erholung sechs Tage in Gardona [sic!]. Ich sah [...] das große Monument, das D’Annunzio seiner knabenhaften Eitelkeit errichtet; was mir darüber erzählt wurde, ist halb lustig, halb traurig. Mussolini hat ihm zwei Millionen Lire pro Jahr zugestanden, damit er den Mund hält und sich an seiner Seite zeigt. Und D’Annunzio hat dies angenommen und verwandelt die alte Villa der Thode in ein absurdes, lächerliches Monument. Der Arme - warum ist er nicht im Krieg gefallen, anstatt als Mumie der eigenen Eitelkeit sich selbst zu überleben!!« (Rolland/Zweig: Briefwechsel 1910-1940, S. 278) Als Karikatur seiner selbst und als Marionette von Mussolini trägt D’Annunzio keines der heroischen Attribute mehr, die sonst für Zweig einen dämonischen Dichter auszeichnen. Stattdessen bestätigt sein Werdegang als Mensch und als Künstler Zweigs tiefe Überzeugung, dass der Schriftsteller einen Fehler begehe, wenn er sich in die Politik einmische. Kein Wunder, dass D'Annunzio in der Welt von Gestern mit keinem Wort bedacht wird. 
D’Annunzios Gedicht für Eleonora Duse zu übersetzen, ${ }^{55}$ auch wenn er gleichzeitig im Kunstwerk-Aufsatz dessen `Ästhetisierung der Politikı verurteilt. Auch Brecht kann sich der Faszination für D’Annunzios Sprache nicht entziehen und übersetzt dessen bekanntestes Gedicht: La pioggia nel pineto. ${ }^{56}$ Über den italienischen Dichter schreibt er privatim: »[E]r war ein Charlatan, aber dieser Charlatan schrieb Hirtengedichte, die kaum vergehen werden. ${ }^{57}$ Trotz der ideologischen Reserven attestiert er Künstlerpersönlichkeiten wie D’Annunzio, George, Kipling und Ezra Pound »eine gewisse feudale Würde $\ll{ }^{58}$ Wie Polgar und Benda plädiert Brecht dafür, das Phänomen D’Annunzio in einem europäischen Kontext zu betrachten. Im Gegensatz zu ihnen hält er jedoch dafür, dass der italienische Dichter und seine >Kollegen` aufgrund ihres Werkes Respekt verdienen: gehören sie in seinen Augen einer >feudalen Vergangenheit an, sind sie doch immerhin Teil einer lebendigen Tradition, die man produktiv fortsetzen könne. ${ }^{59}$

Mit seiner österreichfeindlichen Haltung, seinen Reden für den Kriegseintritt Italiens und danach mit seinem Schulterschluss mit Mussolini hat sich D’Annunzio im deutschsprachigen Raum den Ruf des >ungeliebten Dichters` (Anna Kupka) bzw. des »Johannes der Täufer des Faschismus « ${ }^{60}$ erworben - einen Ruf, der ihn bis heute begleitet. Trotz der Vorbehalte gegen seine Verherrlichung des Krieges und seine Unterstützung des italienischen Faschismus zeigt die Anerkennung seines poetischen Talents durch so unterschiedliche Künstlerpersönlichkeiten wie Zweig, Benjamin und Brecht, dass D’Annunzio für eine Moderne steht, die man mit dem Vorwurf des Protofaschismus nicht einfach diskreditieren kann. Diese widersprüchliche Moderne stellt eine Herausforderung dar - ähnlich wie jene von Filippo Tommaso Marinetti, von Gottfried Benn, von Ernst Jünger, von Ezra Pound oder Louis-Ferdinand Céline; eine Herausforderung, der wir uns heute noch ohne Vorurteile stellen müssen.

D’Annunzio: Die göttliche Eleonora Duse, S. 12-15.

Brecht: Regen in Pinienhain, S. 449f. Vgl. Destro, Die Faszination des Unpolitischen.

Brecht: Arbeitsjournal I (18.7.1942), S. 495.

Brecht: Arbeitsjournal II, S. 764.

Vgl. mit Blick auf das Roman-Fragment Die Geschäfte des Herrn Julius Caesar: Knopf: BrechtHandbuch, S. 382.

60 Andres: Die Tode eines Ungeliebten. 


\section{Literaturverzeichnis}

Andres, Stefan: Die Tode eines Ungeliebten. Eine Annäherung an Gabriele D'Annunzio, den Johannes der Täufer des Faschismus. »Kritische Ausgabe« 2 (2004), S. 19-23.

Altenberg, Peter: Nachfechsung. Berlin: Samuel Fischer Verlag 1916.

Birk, Matjaž; Eicher, Thomas (Hgg.): Stefan Zweig und das Dämonische. Würzburg: Königshausen \& Neumann 2008.

Blei, Franz: Das große Bestiarium der modernen Literatur. Hg. Rolf-Peter Baacke. Hamburg: Europäische Verlagsanstalt 1995.

Brecht, Bertolt: Arbeitsjournal. Bd I: 1928-1942. Hg. Werner Hecht. Frankfurt/M.: Suhrkamp 1973.

Brecht, Arbeitsjournal. Bd II: 1942-1955. Hg. Werner Hecht. Frankfurt/M.: Suhrkamp 1973.

Brecht, Bertolt: Regen in Pinienhain. In: ders.: Gedichte aus dem Nachlass. Gesammelte Werke in 8 Bänden. Hg. Herta Ramthun. Frankfurt/M.: Suhrkamp 1982.

Brittnacher, Hans Richard: »Der Rhythmus hat immer recht."D’Annunzio verhängt den Ausnahmezustand in Fiume. In: Europa neu denken II. Mentalitätsgeschichte der Adria - Neugierde und Konflikt als Betriebsgeheimnis. Hgg. Michael Fischer, Johannes Hahn. Salzburg: Verlag Anton Pustet 2015, S. 47-58.

Corino, Karl: Robert Musil und Italien. Ein Itinerar in Bildern und Texten. Klagenfurt, Wien: Kitab-Verlag 2015.

D’Annunzio, Gabriele: Die göttliche Eleonora Duse. In: Walter Benjamin: Gesammelte Schriften. Supplement I. Kleinere Übersetzungen. Hg. Rolf Tiedemann, Frankfurt/M.: Suhrkamp 1999 (ital. Original: S. 12 u. 14, Benjamins Übersetzung: S. 13 u. 15).

Dangel-Pelloquin, Elsbeth: »Mondaine Stimmungsakrobaten«. Bahrs und Hofmannsthals Kreation der Moderne am Beispiel von Eleonora Duse und Isadora Duncan. In: Hermann Bahr: Österreichischer Kritiker europäischer Avantgarden. Hgg. Martin Anton Müller, Claus Pias, Gottfried Schnödl. Bern u.a.: Lang 2014, S. 51-81.

Demetz, Peter: Die Flugschau von Brescia. Kafka, d'Annunzio und die Männer, die vom Himmel fielen. Wien: Zsolnay 2002.

Destro, Alberto: Die Faszination des Unpolitischen. Brecht übersetzt D’Annunzio. In: Wo bleibt das »Konzept «? Dov' e il »concetto«? Festschrift für/Studi in onore di Enrico De Angelis. Hgg. Carlo Carmassi, Giovanna Cermelli, Marina Foschi Albert, Marianne Hepp. München: iudicium-Verlag 2009, S. 247-255.

Friedell, Egon: Von Dante zu d'Annunzio. Wien, Leipzig: Rosner 1915.

Gec (Enrico Gianeri): D’Annunzio nella caricatura mondiale. Milano: Garzanti 1941.

Ginzkey, Franz Karl: Die Front im Tirol. Berlin: Fischer 1916.

Gumbrecht, Hans Ulrich; Kittler, Friedrich; Siegert, Bernhard (Hgg.): Der Dichter als Kommandant. D’Annunzio erobert Fiume. München: Fink 1996.

Hofmannsthal, Hugo von: Antwort auf die »neunte Canzone "Gabriele D’Annunzios. In: ders.: Gesammelte Werke in Einzelausgaben. Prosa III. Hg. Herbert Steiner. Frankfurt/M.: Fischer 1984, S. 81-86.

Hofmannsthal, Hugo von: D’Annunzio. In: ders.: Gesammelte Werke in zehn Einzelbänden. Reden und Aufsätze I. Hg. Bernd Schoeller. Frankfurt/M.: S. Fischer 1979, S. 174-185.

Ingold, Felix Philipp: Literatur und Aviatik. Europäische Flugdichtung 1909-1927. Basel, Stuttgart: Birkhäuser 1987.

Knopf, Jan: Brecht-Handbuch. Lyrik, Prosa, Schriften. Stuttgart: Metzler 1984.

Koch, Hans-Albrecht: Dante bei Rudolf Borchardt und Hofmannsthal. »Cultura tedesca" 8 (1977), S. 53-67. 
Kraus, Karl: Die letzten Tage der Menschheit. Tragödie in fünf Akten mit Vorspiel und Epilog, Frankfurt/M.: Suhrkamp 1986.

Kraus, Karl: Es wird ernst. »Die Fackel« XIII.343/344 (29.2.1912), S. $44 f$.

Kupka, Anne: Der ungeliebte D’Annunzio. D’Annunzio in der zeitgenössischen und der gegenwärtigen deutschsprachigen Literatur. Frankfurt/M., Bern u.a.: Lang 1992.

Lunzer, Renate: »O poésie, voilà le fruit de tes accouplements avec la politique... ¿D’Annunzio all'avanguardia contro l'Austria. In: Felix Austria - Italia infelix? Tre secoli di relazioni culturali italo-austriache. A cura di Nicoletta Dacrema, postfazione Angelo Ara. Roma: Aracne 2004, S. 104-124.

Lunzer, Renate: Zum Thema »trahison des clercs«. Fallbeispiel Gabriele D’Annunzio. In: Wenn Ränder Mitte werden. Festschrift für Fritz Peter Kirsch. Hg. Chantal Adobati. Wien: WUV 2001, S. 617-626.

Meli, Marco; Ariè Geiger, Elsa (Hgg.): Benno Geiger e la cultura europea. Firenze: Leo Olschki 2010.

Musil, Robert: Tagebücher, Aphorismen, Essays und Reden. In: ders.: Gesammelte Werke in Einzelausgaben. Heft 30. Hg. Adolf Frisé. Reinbek bei Hamburg: Rowohlt 1955.

Polgar, Alfred: d'Annunzio. In: ders.: Kleine Schriften. Bd. I: Musterung. Hg. Marcel ReichRanicki. Reinbek bei Hamburg: Rowohlt 1982, S. 6-10.

Raponi, Elena: Hofmannsthal e l'Italia. Fonti italiane nell'opera poetica e teatrale di Hugo von Hofmannsthal. Milano: Vita e Pensiero Università 2002.

Rauchensteiner, Manfried: Kriegserklärung an Österreich. Das sterbende Kamel. »Die Presse« (22. Mai 2015).

Rauchensteiner, Manfried; Broukal, Josef: Der Erste Weltkrieg und das Ende der Habsburgermonarchie 1914-1918. In aller Kürze. Wien [u.a.]: Böhlau 2015.

Ritter-Santini, Lea: Pari e Impari. Gabriele D’Annunzio e i barbari. In: Italia viva. Studien zur Sprache und Literatur Italiens. Festschrift für Hans Ludwig Scheel. Hgg. Willi Hirdt, Reinhard Klesczewski. Tübingen: Narr 1983, S. 335-351.

Rolland, Romain; Zweig, Stefan: Briefwechsel 1910-1940. Bd. I. Berlin: Rütten \& Leoning 1987.

Schneider, Uwe; Schumann, Andreas (Hgg.): Krieg der Geister. Erster Weltkrieg und literarische Moderne. Würzburg: Königshausen \& Neumann 2000.

Schnitzler, Arthur: Briefe 1913-1931. Bd. II. Hgg. Peter Michael Braunwarth, Richard Miklin, Susanne Peterlik, Heinrich Schnitzler. Frankfurt/M.: Fischer 1984.

Schnitzler, Arthur: Tagebuch 1913-1916. Hg. Werner Welzig. Wien: Verl. d. Österr. Akad. d. Wiss. 1983.

Werfel, Franz: Zwischen Oben und Unten. Prosa, Tagebücher, Aphorismen, literarische Nachträge. München [u.a.]: Langen-Müller 1975.

Zweig, Stefan: Der Kampf mit dem Dämon. Hölderlin, Kleist, Nietzsche. Frankfurt/M.: Fischer 2007.

Zweig, Stefan: Die um Stefan George. »Das litterarische Echo«6.3 (1.11.1903), Sp. 169-172.

Zweig, Stefan: Römische Elegien. Von Gabriele D’Annunzio. »Das literarische Echo« 5.18 (15.7.1904).

Zweig, Stefan: Tagebücher. Hg. Kurt Beck. Frankfurt/M.: S. Fischer 1984.

Zweig, Stefan: Venedigs glückhaftes Schiff. Gabriele d’Annunzios »La Nave«. »Neue Freie Presse« (31.1.1908), S. 1-3.

Zweig, Stefan: Vom neuen Italien. »Neue Freie Presse« (21.7.1908). 